\title{
Resource and Power Costs in Dynamic Spectrum Allocation
}

\author{
Joydeep Acharya, Roy D. Yates \\ Wireless Information Network Laboratory (WINLAB) \\ Rutgers University \\ 671 US Route 1 South, North Brunswick, NJ 08902 \\ \{joy, ryates\}@winlab.rutgers.edu
}

\begin{abstract}
We consider a network setting, where a single Service Provider (SP) provides wireless data services to a group of users in the downlink. The transmission model is similar to OFDMA and thus the SP allocates spectrum to the users. The SP transmits at specific power spectral density. A user applications are characterized by its received rate which is a function of the allocated spectrum, its link gain to the SP and the transmit power spectral density. The SP obtains the net spectrum requested by all the users from a central clearinghouse. The SP charges the users a two part tariff consisting of a fixed subscription fee and variable usage cost and pays the clearinghouse a spectrum cost. The SP also incurs a cost proportional to the power it transmits to all the users. We model this allocation scheme and characterize the trade-off between transmit power spectral density and net spectrum purchased by the SP as a function of the spectrum and power costs for different classes of concave user utilities.
\end{abstract}

\section{INTRODUCTION}

Traditional wireless networks, such as $2 \mathrm{G}$ cellular, allocate fixed spectrum to its customers. Numerous studies have shown that this leads to spectrum wastage and causes artificial spectrum scarcity [1]. Thus dynamic allocation of spectrum have been proposed for better utilization [2], [3]. In the future it is likely that the spectrum regulatory bodies such as FCC will grant a wireless service providers (SP) with short term licenses [4] so that it can purchase the exact amount of spectrum as needed to serve its customers.

Motivated by these developments, we consider a centralized network consisting of a single service provider (SP) that allocates orthogonal chunks of spectrum to its customers dynamically, based on their demand. It then transmits to these users over their allocated spectrum. The user demand of spectrum depend on the received rate which is different for users due to the variations in the link gain. The SP purchases the amount of spectrum needed by its customers, from the FCC. The SP has to pay the FCC for the purchased spectrum and in turn charges the users to recover its costs. In this work we model the dynamic allocation as a SP profit maximization problem and derive the optimal values of the prices.

Pricing for profit maximization has been studied under various contexts. Simple wireless settings have been considered in [5], [6] but the full range of relationships between spectrum prices, costs and user demands are not established. On the other hand, several works in microeconomics have considered pricing for profit maximization [7], [8] but for very generic user demand functions and costs. In this work, we have applied some of these principles specifically to a wireless setting and evaluated the prices and characterized the behavior of the allocation. This is an extension of our previous work [9] where we had considered that the SP has a fixed power $P$ to allocate to the users. Though a standard assumption for power limited devices, in reality the SP will be wired and hence not power limited. A more important practical constraint for wireless settings is the FCC mandated spectral mask i.e. maximum value of transmit power per unit spectrum. Hence in this work we assume that the SP transmits with a spectral density and there is a cost associated with the total transmit power. We have also considered two classes of concave utility functions that model wireless data applications.

Pricing for resource allocation has also been considered in many non-wireless flow control problems [10]. Though some fundamental results stay same across models, our focus is more on capturing the problems specific to the wireless model.

\section{SYSTEM MODEL}

Let there be one SP and $L$ users in the system. The SP transmits to users in the downlink with power spectral density of $\nu$ Watts/MHz. Let the link gain between SP the user $j$ be given by $h_{j}$. The spectral efficiency in the transmission to user $j$ is given by

$$
K_{j}=\log \left(1+\frac{\nu h_{j}}{N_{0}}\right) .
$$

The transmission is similar to OFDMA where different users are allocated different number of tones, as per their application requirements. If the tone spacing is narrow as compared to the total bandwidth, we can assume the frequency variable to be continuous. An example system is LTE which can operate with $15 \mathrm{KHz}$ spacing and 2048 subcarriers [11]. Thus the rate achieved by user $j$ and the transmit power required are

$$
\begin{aligned}
& R_{j}=R\left(\nu, x_{j}\right)=K_{j} x_{j} \\
& P_{j}=P\left(\nu, x_{k}\right)=\nu x_{j} .
\end{aligned}
$$

User $j$ 's application is characterized by a utility function $U_{j}\left(R_{j}\right)$ which is increasing and concave in $R_{j}$. Subsequently we will also allow slight abuse the notation and denote the utility by $U_{j}\left(x_{j}, \nu\right)$. The SP charges a two part tariff [7] from user $j$, consisting of a fixed connection price $\kappa$ and a price $\mu$ charged per unit of spectrum used. This results in a SP revenue of $\rho\left(x_{j}\right)=\mu x_{j}+\kappa$ from user $j$. The user does not have to 
pay the connection price $\kappa$ if he is not receiving any service from the SP, i.e. if $x_{j}=0$. Thus

$$
\rho\left(x_{j}\right)= \begin{cases}\mu x_{j}+\kappa, & x_{j}>0 \\ 0, & x_{j}=0\end{cases}
$$

\section{SPECTRUM Allocation AND PRICING}

The SP initially announces a price pair $(\mu, \kappa)$. Given this, the user $j$ optimizes $x_{j}$ as

$$
\max _{x_{j}} U_{j}\left(x_{j}, \nu\right)-\rho\left(x_{j}\right) .
$$

Note that if the price pair $(\mu, \kappa)$ is high, some of the users may refuse service and hence $x_{j}=0$ for these users. After all users perform this optimization, they inform the SP about how much spectrum they desire. If user $j$ receives non zero spectrum, then

$$
\max _{x_{j}} U_{j}\left(x_{j}, \nu\right)-\mu x_{j}-\kappa>0 .
$$

It can be easily verified that (4) is concave in $x_{j}$. Taking derivatives

$$
\mu=\frac{\partial U\left(x_{j}, \nu\right)}{\partial x_{j}}
$$

The graph of (6) is called the demand function [8] which shows how the demand for resource $x$ varies with price $\mu$.

The SP has to provide a total spectrum of $X=\sum_{j=1}^{L} x_{j}$. It purchases $X$ from the FCC and has to pay $C(X)$. It also incurs a power cost $F(\nu, X)$ for transmitting with $\nu$ Watts/MHz over a bandwidth of $X \mathrm{MHz}$.

The SP maximizes its profits $\Pi=\sum_{j=1}^{L} \rho\left(x_{j}\right)-C(X)-$ $F(\nu, X)$ over prices $(\mu, \kappa)$ for given $\nu$.

$$
\Pi^{*}(\nu)=\max _{\mu, \kappa} \mu X+\kappa L-C(X)-F(\nu, X) .
$$

It then broadcasts the new prices $(\mu, \kappa)$ and the users optimize over $x_{j}$ again.

The optimization problem (4) and (7) is an example of a Stackelberg game [12, Ex. 97.3]. When the SP announces a price tuple $(\mu, \kappa)$, it knows how the user will react and can decide its price accordingly.

\section{A. The Marginal User Principle}

Define the surplus of user $j$ as

$$
S_{j}=U_{j}\left(x_{j}, \nu\right)-\mu x_{j},
$$

which is the residual utility after paying the usage fee. Let the system requirements be such that the SP has to serve all the users. To maximize its profits, the SP will raise its prices $(\mu, \kappa)$ to the point that the surplus of some user $m$ is equal to $\kappa$. After paying the connection fee $\kappa$, user $m$ 's residual utility is zero. If the prices are raised any further, user $m$ will decide not to obtain service from the SP. User $m$ with $S_{m}=\kappa$ is said to be indifferent from obtaining the service [8]. From (4)

$$
\max _{x_{m}} U\left(x_{m}, \nu\right)-\mu x_{m}-\kappa=0 .
$$

From (5), (6), (7) and (9), the complete SP optimization, $\Pi^{*}(\nu)$, is given by

$$
\begin{aligned}
\max _{\mu, \kappa} & \mu X+\kappa L-C(X)-F(\nu, X) \\
\text { s.t. } & \mu=\frac{\partial U\left(x_{j}, \nu\right)}{\partial x_{j}}, 1 \leq j \leq L \\
& \max _{x_{m}} U\left(x_{m}, \nu\right)-\mu x_{m}-\kappa=0, m \in\{1, L\} \\
& \max _{x_{k}} U\left(x_{k}, \nu\right)-\mu x_{k}-\kappa>0 \text { for all } k \neq m .
\end{aligned}
$$

The first order condition of (10a) is

$$
X+L \frac{\partial \kappa}{\partial \mu}+\left(\mu-C^{\prime}(X)-\frac{\partial F}{\partial X}\right) \frac{\partial X}{\partial \mu}=0 .
$$

Let $x_{m}$ be the spectrum allocated to the marginal user. It was shown in [9] that

$$
\partial \kappa / \partial \mu=-x_{m}
$$

To calculate the optimal prices we first define the elasticity of demand, $\epsilon$, [8] which gives the relationship between percentage change in demand to the percentage change in price,

$$
\epsilon=-\frac{\partial X / X}{\partial \mu / \mu}=-\frac{\mu}{X} \frac{\partial X}{\partial \mu}>0 .
$$

The last inequality holds as $\partial X / \partial \mu<0$ as demand reduces with price. Defining $s=x_{m} / X$, the fraction of the spectrum allocated to the marginal user, we can show that the (11) can be re-written to solve for $\mu$ as

$$
C^{\prime}(X)+\frac{\partial F}{\partial X}=\mu\left[1-\frac{1-L s}{\epsilon}\right] .
$$

We now characterize the solution for specific SP cost functions $C(X)$ and $F(\nu, X)$ and user utilities $U(R)$.

\section{B. SP Cost Functions}

The spectrum cost $C(X)$ is the license fees paid by the SPs to the FCC. As mentioned in [9], a reasonable model for cost is $k_{1} k_{2} S X$, where $S$ is base spectrum cost in dollars/MHz. Factor $k_{1}$ denotes the geographical region in which the SP wants to operate as spectrum can be more expensive in urban zones. Factor $k_{2}$ denotes the band in which the spectrum is leased, with a $\mathrm{MHz}$ in the crowded bands such as 800-900 $\mathrm{MHz}$ being more costly than a $\mathrm{MHz}$ in the relatively unused bands. Thus we model $C(X)=C X$.

The SP also pays a power cost proportional to its total transmit power, given by

$$
F(\nu, X)=T \nu X
$$

where $T$ is the constant of proportionality. A part of it could be the electricity costs. The FCC may also levy an cost on transmit power which is additional to the spectrum cost $C$. This is because transmission with a higher power spectral density $\nu$ could cause interference to other systems potentially operating in that band. A possible example could be that the SP considered is a 802.22 transmitter [13], a secondary system operating in the TV bands. To safeguard the primary TV transmitters, the FCC may decide to charge the 802.22 SP more if it transmits with more power. 
Note that for these cost functions the SP profit in (10a) becomes

$$
\Pi^{*}(\nu)=\max _{\mu, \kappa}(\mu-C-T \nu) X+\kappa L
$$

We introduce the following notation

$$
C_{e}=C+T \nu,
$$

which we will call as the effective spectrum cost.

\section{Logarithmic User Utilities}

These utilities, given by $U_{j}\left(R_{j}\right)=\log \left(1+R_{j}\right)=\log (1+$ $\left.K_{j} x_{j}\right)$, are used to model elastic applications like data. For user $j$, the demand function in (6) is given by

$$
\mu=\frac{K_{j}}{1+K_{j} x_{j}} .
$$

The maximum value of the RHS of (18) is $K_{j}$ (for $x_{j}=0$ ) and thus for a feasible allocation,

$$
\mu<\min _{j} K_{j}=K_{m} .
$$

A plot of the demand function is given in Figure (1).

Lemma 1: For logarithmic utilities, the user with the weakest link gain to the SP is the marginal user.

Proof: Let the optimal value of the spectrum price be $\mu^{*}$. The marginal user is the user who has the least surplus. Substituting for $x_{j}^{*}$ from (18) in (8), the surplus of user $j$ is given by

$$
S_{j}=\log \left(\frac{K_{j}}{\mu^{*}}\right)-1+\frac{\mu^{*}}{K_{j}} .
$$

Taking derivatives of $S_{j}$ w.r.t. $K_{j}$ and using relation (19), it can be shown that $\partial S_{j} / \partial K_{j}>0$ i.e. $S_{j}$ increases in $K_{j}$. So the user, $m$ with least surplus $S_{m}$ is given by $m=$ $\arg \min _{j} K_{j} \stackrel{(a)}{=} \arg \min _{j} h_{j}$. Relation (a) follows from (1).

A graphical proof for a similar system was given in [9]. Substituting for $x_{j}$ from (18) in (14) and after some algebraic manipulation, we can show that the optimal value of the price $\mu=\mu^{*}$ satisfies the following quadratic equation

$$
\begin{aligned}
& \quad\left(\frac{1}{K_{m}}-\frac{K_{s}}{L}\right) \mu^{2}-\mu+C_{e}=\left.0\right|_{\mu=\mu^{*}} \\
& \text { where } K_{s}=\sum_{j=1}^{L} \frac{1}{K_{j}} .
\end{aligned}
$$

The optimal values of spectrum, $x_{j}^{*}$ are given by substituting for $\mu=\mu^{*}$ in (18). Denote $X^{*}=\sum_{j} x_{j}^{*}$.

Lemma 2: For users with logarithmic utilities, the spectrum is overpriced, i.e. $\mu^{*}>C_{e}$.

Proof: Relation (21a) can be rewritten as

$$
\left(\frac{1}{K_{m}}-\frac{K_{s}}{L}\right) \mu^{* 2}=\mu^{*}-C_{e} .
$$

Using (19), we can prove that $\left(1 / K_{m}-K_{s} / L\right)>0$ and hence the LHS of (22) is positive. Thus the RHS has to be positive which yields the desired result.
An intuitive explanation is that, the logarithmic utility function is unbounded from above and there is always demand even for high prices. So the SP exploits this to maximize his profits by keeping $\mu$ above $C_{e}$. However this does not mean that the SP can arbitrarily overprice spectrum. To understand this, we first calculate the value of the elasticity, $\epsilon^{*}$ for the given optimal values of $X^{*}$ and $\mu^{*}$. Using the value of $X^{*}$ from (18) in (13) we can show that

$$
\epsilon^{*}=\frac{L}{L-\mu^{*} K_{s}}>1
$$

Lemma 3: For logarithmic utilities, the aggregate demand function of spectrum for all users is elastic.

Proof: This follows from (23) [8, Chapter 15].

For elastic demands, the percentage change in spectrum demanded is greater than that percentage change in price. Hence, when the optimal price $\mu^{*}$ is increased, percentage decrease in spectrum demand is higher and the total revenue of the SP given by $\mu^{*} X^{*}$ decreases.

Now let us look at the SP profit. From (16)

$$
\Pi^{*}\left(\nu, \mu^{*}\right)=\underbrace{\left(\mu^{*}-C_{e}\right) X^{*}}_{\Pi_{U}^{*}}+\underbrace{\kappa^{*} L}_{\Pi_{C}^{*}} .
$$

Note that $\Pi_{U}^{*}$ and $\Pi_{C}^{*}$ are the profits from the usage cost and the subscription fees respectively. We now want to investigate how $\Pi^{*}\left(\nu, \mu^{*}\right)$ changes as a function of spectrum price $\mu^{*}$.

Lemma 4: The following results hold about the SP profit functions.

a) The profit from subscription, $\Pi_{C}^{*}$ decreases with cost $\mu^{*}$.

b) The profit from usage, $\Pi_{U}^{*}$ increases with $\mu^{*}$ for $C_{e}<$ $\mu^{*}<\sqrt{C_{e} L / K_{s}}$

Proof: Refer to Appendix I.

The fact that there is a maximum threshold on $\mu^{*}$, occurs because of the elastic nature of spectrum demand, i.e. the increase in $\mu^{*}$ is more than offset by the decrease in $X^{*}$.

\section{Exponential User Utilities}

Such utilities given by, $U_{j}\left(R_{j}\right)=\Gamma_{j}\left(1-e^{-R_{j} / \Gamma_{j}}\right)=$ $\Gamma_{j}\left(1-e^{-\left(K_{j} / \Gamma_{j}\right) x_{j}}\right)$, model situations where the user $j$ has a target rate $\Gamma_{j}$. From (6), the demand function of user $j$ is given by

$$
\mu=K_{j} e^{-\left(K_{j} / \Gamma_{j}\right) x_{j}} .
$$

This is shown in Figure 1. Being of exponential dependence, the demand functions decreases more sharply than the logarithmic demand function of (18). This is a consequence of the fact the logarithmic utilities are unbounded from above and there is always demand for spectrum, unlike the exponential utilities, which flatten at a value of $\Gamma_{j}$ for high spectrum $x_{j}$ and in that regime there is little demand for spectrum.

Lemma 5: For exponential utilities, when all users have equal target rates, the user with the weakest link gain to the $\mathrm{SP}$ is the marginal user.

Proof: Let $\Gamma_{j}=\Gamma$ for all users $j$. Substitute for $x_{j}^{*}$ from (25) in expression of user surplus in (8) to obtain,

$$
S_{j}=\Gamma\left(1-\frac{\mu^{*}}{K_{j}}\right)-\mu^{*} \frac{\Gamma}{K_{j}} \log \left(\frac{K_{j}}{\mu^{*}}\right) .
$$



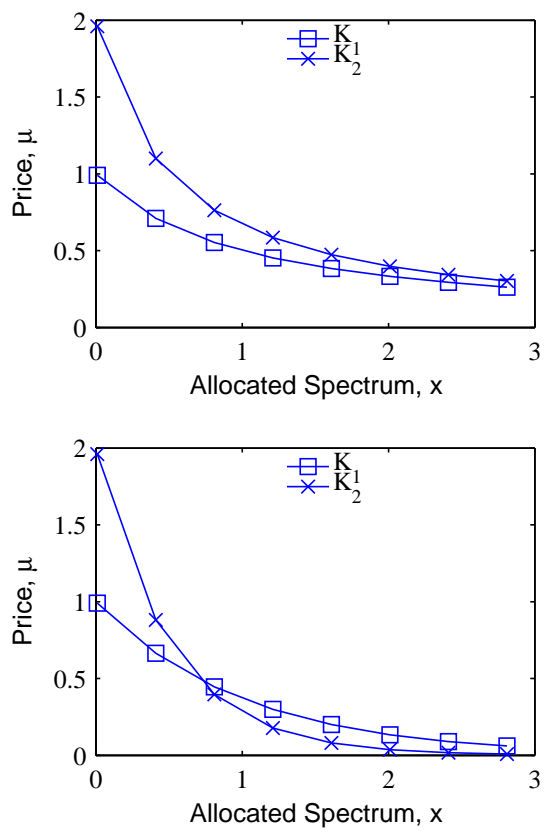

Fig. 1. Demand functions for logarithmic (top) and exponential utilities (bottom) with two users with spectral efficiencies $K_{1}=1$ and $K_{2}=2$ and $\Gamma=1$ for the exponential utility target rate

Similar to proof in Lemma 1 we can show that $\partial S_{j} / \partial K_{j}>0$ and the remainder follows.

Note that relation (19) also holds for exponential utilities.

Lemma 6: The following facts hold

a) For very low prices, $\mu \sim 0$, the marginal user obtains the maximum spectrum.

b) For high prices, $\mu \sim K_{m}$, (from (19)) the marginal user obtains the least spectrum.

While we omit proof due to space considerations, we observe that

a) For low $\mu^{*}$, enough spectrum can be bought by each user $j$ to make $R_{j}$ high enough such that $U_{j}\left(R_{j}\right) \rightarrow \Gamma$. But user $m$ with weakest $K_{m}$ would have to purchase more spectrum than others to reach to a high $R_{m}$ (as $\left.R_{m}=K_{m} x_{m}\right)$. Note that this purchase is possible as $\mu^{*}$ is low and any amount of spectrum can be bought.

b) Looking at (25) for marginal user $m$, we see that when $\mu^{*} \rightarrow K_{m}$, the spectrum $x_{m}^{*} \rightarrow 0$.

Contrast Lemma 6 with the the logarithmic utility case when the marginal user always obtained the least spectrum.

Substituting for $x_{j}$ from (25) in (14) and after some algebraic manipulation, we can show that the price $\mu$ is given by the positive solution of

$$
\begin{aligned}
C_{e} & =\left(1-C_{1}\right) \mu+C_{2} \mu \log (\mu), \text { where } \\
C_{1} & =\left(\sum_{j=l}^{L} \frac{\Gamma_{j}}{K_{j}}\right)^{-1}\left[\sum_{j=l}^{L} \frac{\Gamma_{j}}{K_{j}} \log \left(K_{j}\right)-\frac{L \Gamma_{m}}{K_{m}} \log \left(K_{m}\right)\right] \\
C_{2} & =\left(\sum_{j=l}^{L} \frac{\Gamma_{j}}{K_{j}}\right)^{-1}\left[\sum_{j=l}^{L} \frac{\Gamma_{j}}{K_{j}}-\frac{L \Gamma_{m}}{K_{m}}\right]
\end{aligned}
$$

We now show that unlike the logarithmic utility case, the exponential utilities do not always overprice spectrum.

Lemma 7: If $C_{1}>1$, then for users with exponential utilities having the same target rates, the optimal price, $\mu^{*}<1$. Thus the spectrum is underpriced, i.e. $\mu^{*}<C_{e}$ when $C_{e}>1$.

Proof: Refer to Appendix II.

To calculate the elasticity, substitute for $x_{j}^{*}$ from (25) in (13)

$$
\epsilon^{*}=\frac{\sum_{j=1}^{L} \frac{1}{K_{j}}}{\sum_{j=1}^{L} \frac{1}{K_{j}} \log \left(\frac{K_{j}}{\mu^{*}}\right)} .
$$

Note that $\epsilon^{*} \ngtr 1$ for all cases unlike the logarithmic utility case in (23).

Lemma 8: For exponential utilities, the aggregate demand function of spectrum is inelastic, i.e. $\epsilon^{*}<1$ when

$$
\mu^{*}<\mu_{0}=\frac{K_{m}}{e} .
$$

Proof: The $j^{\text {th }}$ term in numerator of $\epsilon^{*}$ is multiplied by $\log \left(K_{j} / \mu^{*}\right)$ in the corresponding term of the denominator. We have to find conditions for $\epsilon^{*}<1$. One straightforward condition is $\log \left(K_{j} / \mu^{*}\right)>1$ for all $j$. This implies $\mu^{*}<$ $K_{j} / e$ for all $j$. The rest follows from (19).

At lower values of prices, characterized by being below threshold $\mu_{0}$, each user has adequate spectrum to be in the flat region of the exponential utility. Even if price $\mu^{*}$ changes, users have little incentive to alter their purchased spectrum.

Lemma 9: The following results hold about the SP profit functions.

a) The profit from subscription, $\Pi_{C}^{*}$ decreases with cost $\mu^{*}$.

b) The usage profit, $\Pi_{U}^{*}$ increases when $\mu_{0}>\mu^{*}>C_{e}$.

Proof: Refer to Appendix III.

\section{Numerical Results}

We consider a linear network with one SP and $L=10$ users. For path loss, we choose the COST-231 propagation model [14], at an operating frequency of $2.4 \mathrm{GHz}$. Let the distance of user $j$ from the SP be $d_{j}$. Thus the link gain is given by

$$
h_{j, d B}=-31.5-35 \log \left(d_{j}\right) .
$$

Consider an user arrangement where the vector of user distances are $\mathbf{d}^{(1)}=\left[d_{1}, \cdots, d_{L}\right]=[10,20, \cdots, 10 L]$. We consider users with exponential utilities having $\Gamma_{j}=1 \mathrm{Mbps}$ because the exponential utility allocation results present more 
possible variations as seen in Section III-D. The total SP profit is given in Figure (2) when the power cost is 10 times the total transmit power. We see that the profit reduces with spectrum cost $C$. Also when cost $C$ increases, the SP has an incentive to switch to higher transmit power spectral density $\nu$ as the effective cost $C_{e}=C+T \nu$ is dominated by $C$ and is invariant of $\nu$, but the user utilities and hence payments increase with $\nu$. If $C$ is above a threshold, then the profits reduce to zero as no spectrum is purchased. Similarly for low $C$ regimes, SP cost is dominated by $T \eta$ and the SP has no incentive to transmit at high $\nu$. Though the results in Figure (2) are for $\mathbf{d}^{(1)}$, the general trends hold for other user placements.

Figure (3) plots the SP effective cost $C_{e}$ and spectrum price $\mu$ together. We see that for most portions the spectrum is underpriced. The effect of this is also seen in Figure (4) which plots the breakup of the usage and the connection profits, $\Pi_{U}^{*}$ and $\Pi_{C}^{*}$ respectively. We see the the places where spectrum is underpriced, $\Pi_{U}^{*}$ is a loss. Another thing to note is that the SP profit comes predominantly from the connection fee. These effects are also described in [15]. The intuition is to look at the demand function in (25). Since it extends to infinity, there is a demand even at large amounts of spectrum. However the demand decays exponentially. So the users want a large amount of spectrum but have low willingness to pay usage fees for large amounts. So the SP can't hope to gain from the usage fees. It thus reduces the spectrum price (underpricing it in the process) so that users purchase a lot of spectrum and the SP can makes use of their increased utility by extracting their increased surplus as the connection fee.

Lastly we consider that the SP can operate with $10 \%$ user outage. For $L=10$ users, let link gains satisfy $h_{1}>\cdots>$ $h_{10}$. Thus user 10 is the marginal user. The SP can choose to serve 9 users by raising prices to make user 10 refuse service. User 9 would be the new marginal user. The loss of revenue from user 10 can be made up by the increased revenue from the other users. The results are shown in Figure (5). For high values of $\nu$, the profits are more for $L=10$. For low $\nu$, it is slightly advantageous to serve 9 users when $C$ is high. Recall from Figure (3) that in that regime, $\mu \sim C_{e}$ and the profits are mostly due to the connection fees. So the deciding factor is the relative differences in the surpluses of users 10 and 9.

\section{CONCLUSION}

In this work, we have considered a network where a single service provider allocates spectrum to it's customers in the downlink. We propose a dynamic allocation scheme based on SP profit maximization. The SP uses two part monopolistic pricing, consisting of a fixed connection fee and a variable usage cost. We showed that for a broad range of concave user utilities, the user with the weakest link gain decided the connection fee. We characterized the spectrum allocation and derived values for various prices involved. We showed that for logarithmic utilities, the spectrum was overpriced relative to the costs of the SP and the demand was elastic. In contrast, for users whose applications have exponential utilities, the demand could be inelastic. Numerically we illustrated some key analytical ideas and also tested the performance of the

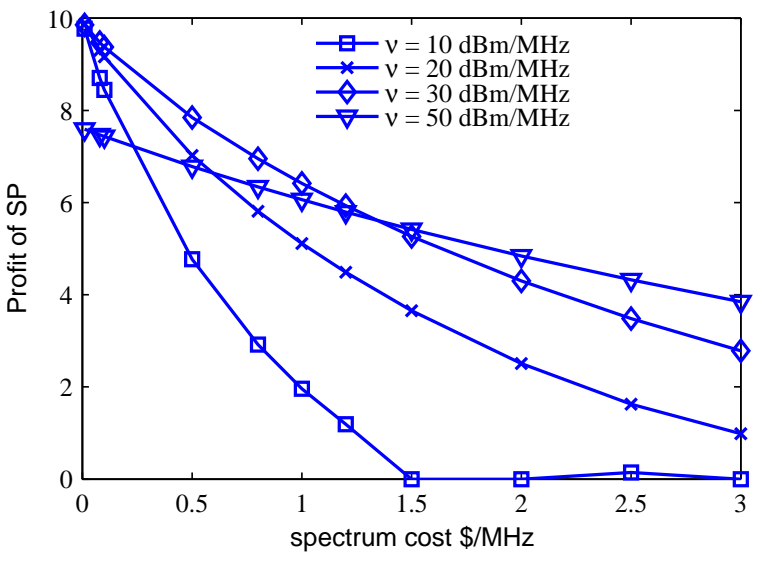

Fig. 2. Total SP profit as function of efficiency and spectrum costs user when each user is homogeneous and has exponential utility with $\Gamma=1 \mathrm{Mbps}$ and $T=10$

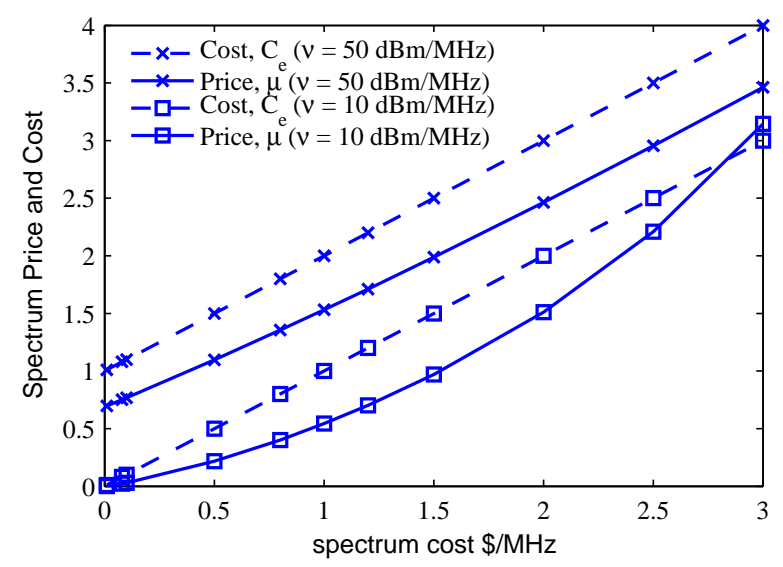

Fig. 3. The effective cost $C_{e}=C+T \nu$ and spectrum price $\mu$ as a function of spectrum cost $C$ when each user is homogeneous and has exponential utility with $\Gamma=1 \mathrm{Mbps}$ and $T=10$

allocation algorithm with user outage. We conclude that the microeconomic model gave us an instructive framework to study the profit maximization problem.

\section{APPENDIX I}

PROOF OF LEMMA 4

a) From (12) we have $\partial \Pi_{C}^{*} / \partial \mu^{*}=-L x_{m}^{*}<0$.

b) Taking derivatives

$\frac{\partial \Pi_{U}^{*}}{\partial \mu^{*}}=X^{*}+\left(\mu-C_{e}\right) \frac{\partial X^{*}}{\partial \mu^{*}} \stackrel{(a)}{=} X^{*}\left[1-\epsilon^{*}\left(\frac{\mu^{*}-C_{e}}{\mu^{*}}\right)\right]$,

where equality $(a)$ follows from (13). From (31), it can be shown $\partial \Pi_{U}^{*} / \partial \mu^{*}>0$ holds when

$$
\epsilon^{*}\left(\frac{\mu^{*}-C_{e}}{\mu^{*}}\right)<1
$$

Substitute for $\epsilon^{*}$ from (23) in (32) and after some manipulations we obtain $\mu^{*}<\sqrt{C_{e} L / K_{s}}$. 


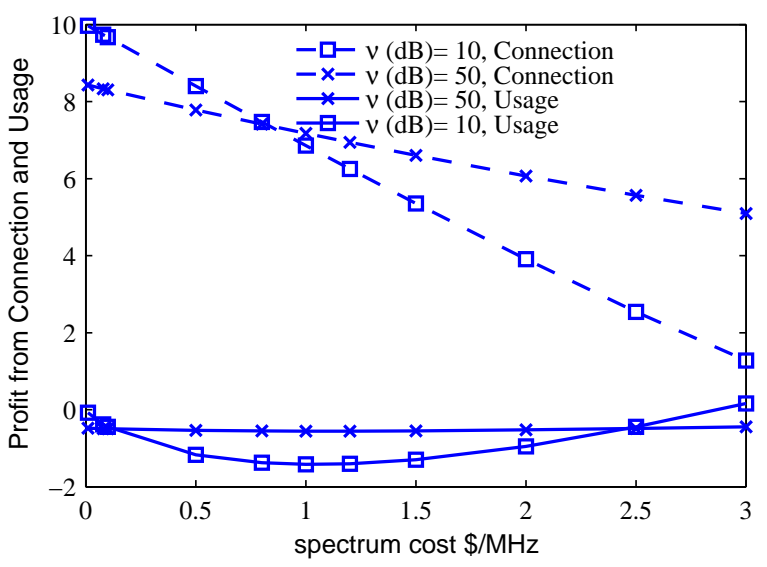

Fig. 4. Breakup of SP profit, $\Pi^{*}$ as total usage cost, $\Pi_{U}^{*}$ and total connection fee, $\Pi_{C}^{*}$ as a function of efficiency and spectrum costs user when each user is homogeneous and has exponential utility with $\Gamma=1$ Mbps and $T=10$

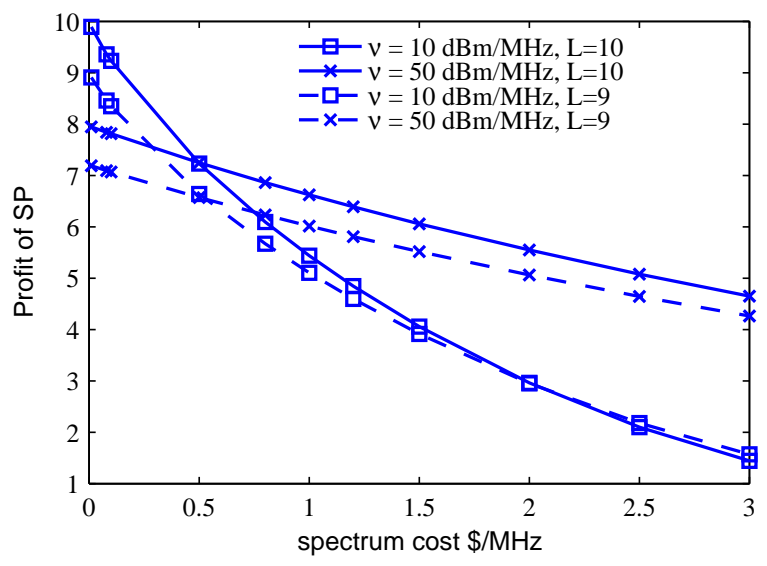

Fig. 5. A example plot of the SP profits for $L=10$ users and with $L=9$ obtained by removing the marginal user from the original population.

\section{APPENDIX II}

PROOF OF LEMMA 7

Equation (27a) can be re-written as

$$
C_{e}+\left(C_{1}-1\right) \mu^{*}=C_{2} \mu^{*} \log \left(\mu^{*}\right)
$$

Thus $\mu^{*}$ is the point of intersection of $h(\mu)=C_{e}+\left(C_{1}-1\right) \mu$ and $g(\mu)=C_{2} \mu \log (\mu)$. Let $\Gamma_{j}=\Gamma$ for all users $j$. From (19) the term in (27c), $C_{2}<0$. When $C_{1}>1, h(\mu)$ is a straight line with a positive slope and intercept. It can be verified that $g(\mu)$ is a concave function with zeros in $\mu=0,1$ and $g(\mu)>0$ for $\mu \in[0,1]$. A plot of the two graphs is shown in Figure (6). It can be seen that the solution, $\mu^{*}<1$.

\section{APPENDIX III \\ PROOF OF LEMMA 9}

a) Proof is same as proof of Lemma 4a)

b) Similar to the proof of Lemma 4b), we have to show that the elasticity satisfies condition (32). Now $\left(\mu^{*}-\right.$

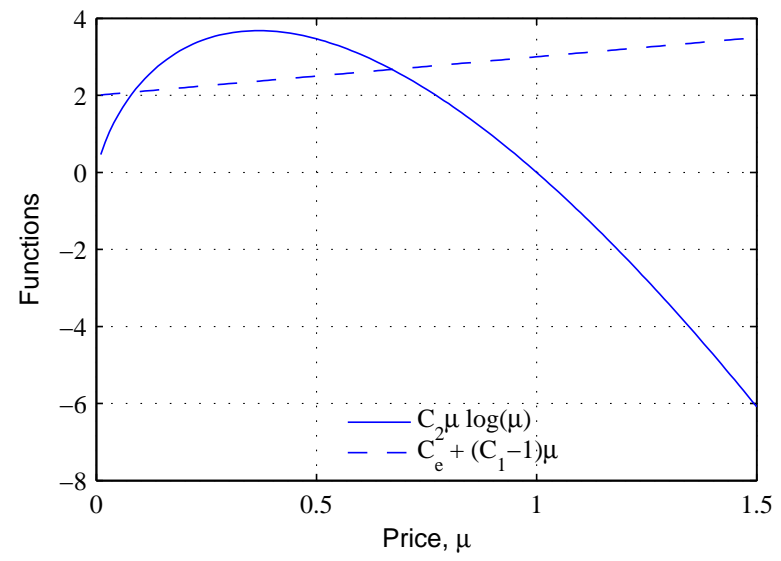

Fig. 6. A example plot of the functions, $h(\mu)=C_{e}+\left(C_{1}-1\right) \mu$ and $g(\mu)=C_{2} \mu \log (\mu)$ when $C_{1}>1$

$\left.C_{e}\right) / \mu^{*}<1$. And it was proved in Lemma 8 that for $\mu^{*}<\mu_{0}$, elasticity, $\epsilon^{*}<1$.

\section{REFERENCES}

[1] M. McHenry and D. McCloskey, "New York City spectrum occupancy measurements, September 2004," no. Available online at: www.sharedspectrum.com/inc/content/measurements/nsf/NYCreport.pdf.

[2] M. Buddhikot and K. Ryan, "Spectrum management in coordinated dynamic spectrum access based cellular networks," In Proc. of IEEE Symposium on New Frontiers in Dynamic Spectrum Access Networks (DYSPAN), pp. 299-307, Nov 2005.

[3] D. P. Satapathy and J. M. Peha, "Spectrum sharing without licenses:opportunities and dangers," Interconnection and the Internet, book of Selected Papers From The 1996 Telecommunications Policy Research Conference, vol. G. Rosston and D. Waterman (Eds.), NJ.

[4] O. Ileri and N. B. Mandayam, "Dynamic spectrum access models: Towards an engineering perspective in the spectrum debate," IEEE Communications Magazine, January 2008.

[5] O. Ileri, D. Samardzija, T. Sizer, and N. B. Mandayam, "Demand responsive pricing and competitive spectrum allocation via a spectrum server," In Proc. of IEEE Symposium on New Frontiers in Dynamic Spectrum Access Networks (DYSPAN), pp. 194-202, Nov 2005.

[6] V. Rodriguez, K. Moessner, and R. Tafazolli, "Market-driven dynamic spectrum allocation: Optimal end-user pricing and admission control for CDMA," in 14th European Information Society Technologies (IST) Mobile and Wireless Communications Summit, 2005, dresden, Germany.

[7] W. Y. Oi, "A disneyland dilemma: Two-part tariffs for a mickey mouse monopoly," The Quarterly Journal of Economics, February 1971.

[8] H. R. Varian, Intermediate Microeconomics. W. W. Norton, 1999.

[9] J. Acharya and R. D. Yates, "Profit maximizing pricing strategies for dynamic spectrum allocation," In Proc. of IEEE Conf. on Information Sciences and Systems (CISS), March 2007.

[10] A. Ozdaglar and R. Srikant, Incentives and Pricing in Communication Networks, chapter in Algorithmic Game Theory. N. Nisan, T. Roughgarden, E. Tardos, and V. Vazirani, editors: Cambridge University Press.

[11] "Long Term Evolution (LTE): Overview of LTE Air Interface," http: //www.motorola.com/mot/doc/6/6993_MotDoc.pdf.

[12] M. J. Osborne and A. Rubinstein, A Course in Game Theory. The MIT Press, 2002.

[13] G. Chouinard, "Wireless regional area network, (WRAN) initial system concept," IEEE 802.22-04-0003-00-0000, vol. Plenary meeting of the IEEE 802.22 WG on WRANs, no. Available online at: http://ieee802.org/22/, Nov 2004.

[14] "Mobile WiMAX part I: A technical overview and performance evaluation, WiMAX forum," http://www.wimaxforum.org/technology/ downloads/Mobile_WiMAX_Part1_Overview_and_Performance.pdf.

[15] J. K. MacKie-Mason and H. R. Varian, "Pricing congestible network resources," IEEE Journal on Selected Areas in Communications, vol. 13, pp. 1141-1149, September 1995. 